\title{
NSTX PLASMA START-UP USING TRANSIENT COAXIAL HELICITY INJECTION
}

\author{
D. Mueller ${ }^{1}$, R. Raman $^{2}$, M.G. Bell ${ }^{1}$, T.R. Jarboe ${ }^{2}$, B. LeBlanc ${ }^{1}$, R. Maqueda ${ }^{3}$, S. Sabbagh ${ }^{4}$, and B.A. Nelson ${ }^{2}$ \\ ${ }^{1}$ PPPL, Princeton, NJ 08543,dmueller@pppl.gov \\ ${ }^{2}$ University of Washington, Seattle, WA 98195 \\ ${ }^{3}$ Nova Photonics, Princeton, NJ 08543 \\ ${ }^{4}$ Columbia Univ, New York, NY 10027
}

Future toroidal magnetic confinement fusion plasma devices such as the Component Test Facility (CTF) require non-inductive toroidal current drive. A new method of non-inductive startup, referred to as transient coaxial helicity injection (Transient CHI), has been developed on the Helicity Injected Torus (HIT-II) experiment and the National Spherical Torus Experiment (NSTX). In this method, plasma current is produced by discharging a capacitor bank between coaxial electrodes in the presence of toroidal and poloidal magnetic fields chosen such that the plasma rapidly expands into the chamber. When the injected current is rapidly decreased, magnetic reconnection occurs near the injection electrodes with the toroidal plasma current forming closed flux surfaces. In NSTX, transient CHI has demonstrated closed-flux current generation of up to 160 $k A$, without the use of a central solenoid. Detailed experimental measurements made on NSTX include fast time-scale visible imaging of the entire process.

\section{INTRODUCTION}

The spherical torus (ST) ${ }^{1}$ concept has advantages as a result of its low ratio of plasma major radius to minor radius, which allows a high ratio of plasma pressure to toroidal field pressure $(\beta)$ and a high fraction of bootstrap current. As a result of this low-aspect ratio, it is important for the next generation of ST experiments, and essential for an ST reactor, that the central solenoid be replaced by other means of plasma current drive. Noninductive means to initiate and maintain a plasma discharge are, therefore, very important.

The National Spherical Torus Experiment (NSTX) is investigating the use of coaxial helicity injection $(\mathrm{CHI})^{2}$ as a method to produce the initial plasma and sufficient toroidal current to provide a target that is sufficient for other forms of non-inductive current drive to ramp-up and maintain the toroidal plasma current.

Fig. 1 shows a cross-sectional view of NSTX. The stainless steel vacuum vessel (major radius $0.85 \mathrm{~m}$ ) is electrically separated into inner and outer sections by means of toroidal ceramic rings at the top and bottom of the center column such that the inner divertor is isolated from the outer diverter. The plasma facing surfaces that are close to the plasma, including the divertor plates, are covered with graphite tiles. Refs. 3 and 4 describe the NSTX device in more detail and present recent experimental results respectively.

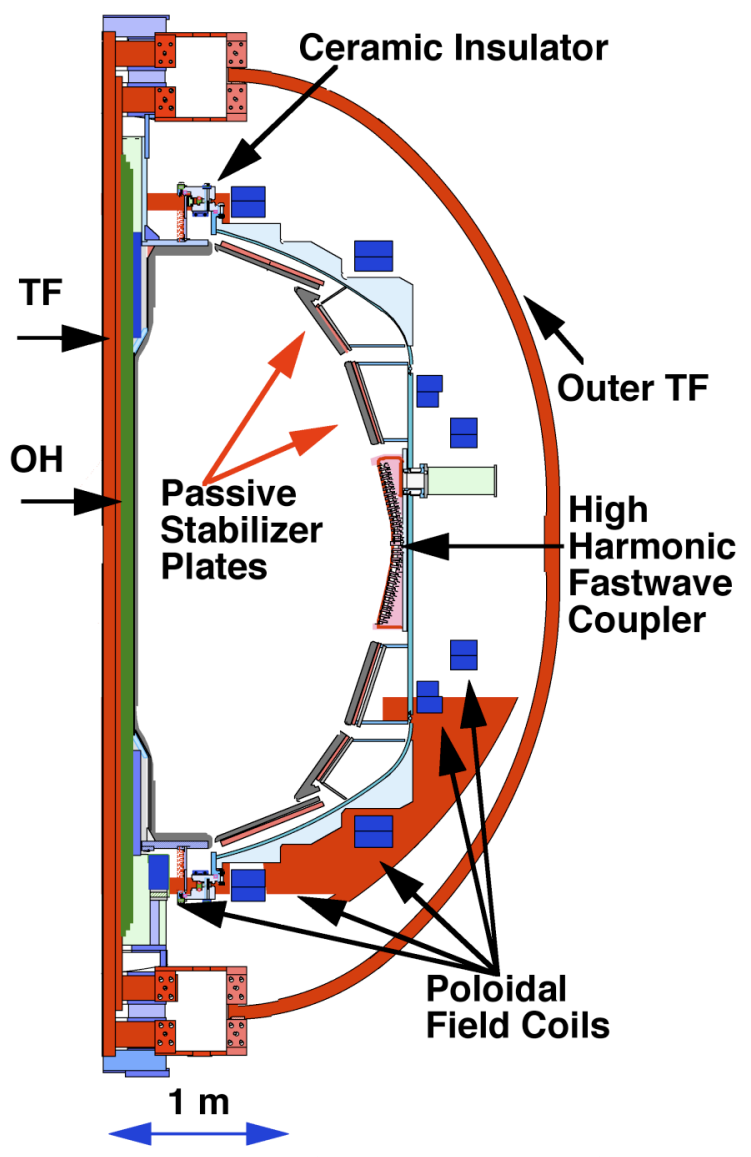

Fig. 1. Cross-sectional view of NSTX. The major radius of the vessel is $0.85 \mathrm{~m}$, the interior height is $3.2 \mathrm{~m}$ 
CHI discharges are initiated by first providing toroidal field and an initial poloidal field that connects the inner and outer divertors as shown schematically in Fig. 2. When gas is introduced into the volume below the lower divertor gap and a voltage ( 1 to $2 \mathrm{kV}$ ) is applied across the gap, a discharge forms and current flows from the outer to the inner divertor plates. The current essentially follows the helical field lines. When the discharge first forms, the plasma is near the divertor gap, which we call the injector region. The region at the top of the machine is referred to as the absorber. As the injector current $\left(\mathrm{I}_{\text {inj }}\right)$ linking the inner and outer divertors increases, the $\mathrm{J}_{\mathrm{pol}} \times \mathrm{B}_{\mathrm{T}}$ force overcomes the field line tension and the plasma expands up into the main chamber as shown in the fast camera frames in Fig. 2, until it fills the torus volume. The top frame in Fig. 2 shows the plasma after $I_{i n j}$ is reduced to zero, after which, the measured toroidal current, $\mathrm{I}_{\mathrm{p}}$, decays to zero with a decay time constant of approximately $5 \mathrm{~ms}$.

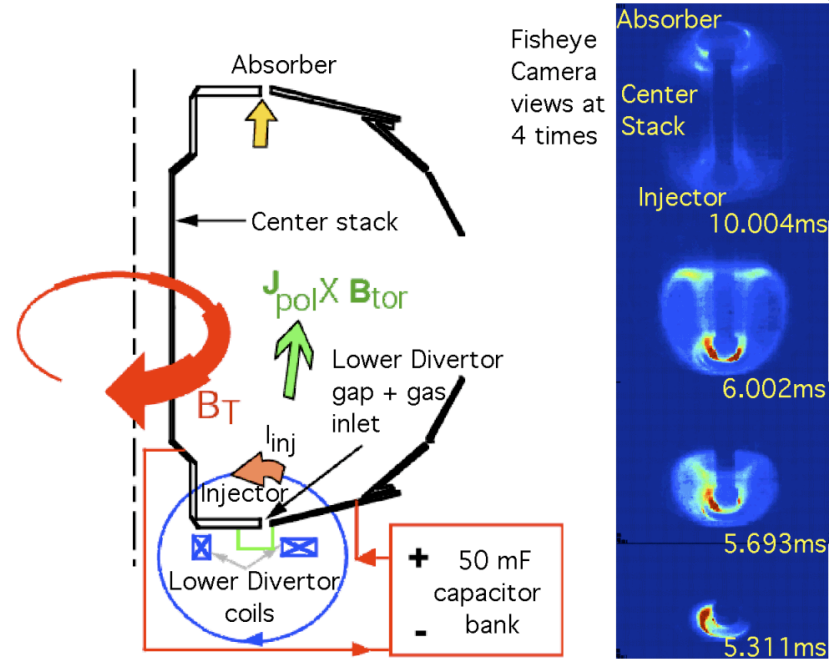

Fig. 2. The schematic diagram shows the CHI start-up configuration on the left. On the right are shown fast TV images of the plasma evolution. Notes: the first light in the injector region was visible after $5.15 \mathrm{~ms}$ and the image from $10.004 \mathrm{~ms}$ is after the injector current returned to zero.

\section{TRANSIENT CHI}

The first CHI experiments on NSTX $^{5}$ attempted to reproduce the results observed on HIT-I ${ }^{6}$ and HIT-II ${ }^{7}$ wherein non-axisymmetric magnetic reconnection and relaxation were employed to initiate and sustain a plasma discharge. These experiments used programmable rectifier power supplies to supply the injector voltage $\left(\mathrm{V}_{\text {inj }}\right)$ between the inner and outer vessel sections. Up to $400 \mathrm{kA}$ of toroidal current was produced in these "steadystate" CHI discharges in NSTX where the resistive decay time scale of the plasma was much less than the discharge duration. There was little evidence that the plasma formed on closed field lines; the discharges generally terminated early due to arcs before the planned end of the discharge; and the high power (up to $30 \mathrm{MW}$ ) used to drive the CHI discharge was not compatible with the power handling capability of the plasma armor for long times. Instead a new technique, called transient $\mathrm{CHI}^{8}$, was developed on HIT-II, to provide a plasma suitable for $I_{p}$ sustainment or ramp-up by other means. In this technique the application of $\mathrm{V}_{\text {inj }}$ is of short duration, typically less than $4 \mathrm{~ms}$, so that $\mathrm{I}_{\mathrm{inj}}$ is forced to zero quickly after formation of the discharge. In this method the initial poloidal field configuration is chosen such that the plasma rapidly expands from the injector region into the main chamber.

There are several requirements to make transient $\mathrm{CHI}$ work.First, there must be enough energy in the voltage supply to provide enough $\mathrm{I}_{\text {inj }}$ to overcome the field line tension and exceed the "bubble burst" condition $I_{i n j}=2 \psi_{i n j}^{2} /\left(\mu_{o}^{2} d^{2} I_{T F}\right)$ where $\mathrm{I}_{\mathrm{TF}}$ is the total toroidal field current through the center of the torus, $d$ is the width of the injector flux footprint on the electrodes and $\psi_{\text {inj }}$ is the poloidal flux linking the injector insulating gap. ${ }^{9}$

Second, the CHI discharge must fill the vessel quickly to keep required duration of $V_{\text {inj }}$ short. This is dependent upon the applied $V_{\text {inj }}$ since this determines the rate at which toroidal flux $\left(\phi_{\mathrm{T}}\right)$ crosses the injector and absorber gaps: $\mathrm{V}_{\text {inj }}=\mathrm{d} \phi_{\mathrm{T}} / \mathrm{dt}$. In NSTX with $\mathrm{B}_{\mathrm{T}}=0.3 \mathrm{~T}$ on axis, $\phi_{\mathrm{T}} \sim 1.4 \mathrm{~Wb}$ inside the vessel. For $2 \mathrm{kV}$, the time needed to replace all the $\phi_{\text {T }}$ within the vacuum vessel is about $0.7 \mathrm{~ms}$. This pulse duration is easily supplied by the capacitor bank for NSTX.

Third, the energy stored in the capacitor bank must be enough to fully ionize and heat the injected gas. It requires about $50 \mathrm{eV}$ to ionize each atom and an additional $60 \mathrm{eV}$ to heat each ion to $20 \mathrm{eV}$. In order to reduce the quantity of gas required to initiate $\mathrm{CHI}$, it was necessary to move the $\mathrm{CHI}$ gas injection port from the main chamber to the small, enclosed volume $(\sim 1001)$ below the lower divertor gap. Furthermore, $10 \mathrm{~kW}$ of 18 $\mathrm{GHz}$ microwave power was injected into this cavity to preionize the gas. Together these changes reduced the total gas needed to achieve breakdown and the energy needed to ionize and heat the gas was lowered to a few $\mathrm{kJ}$, less than is available from the capacitor bank.

Fourth, the capacitor must be able to supply the magnetic energy due to the toroidal plasma current, $1 / 2 L_{p} I_{p}^{2}$, where $\mathrm{L}_{\mathrm{p}}$ is the inductance of the plasma. Since $E_{c a p}=1 / 2 C V^{2}$ and the inductance of typical NSTX plasmas with closed flux surfaces is about $0.5 \mu \mathrm{H}$, this limits $\mathrm{I}_{\mathrm{p}}$ to about $500 \mathrm{kA}$ with the present capacitor bank.

Fifth, the flux footprint of the initial CHI plasma must be narrow enough to make the distance over which 
reconnection needs to occur small, but large enough to be consistent with meeting the "bubble burst" condition.

Finally, applying high voltage to the vacuum vessel raises the possibility of both internal arcs across the insulators and external arcs from the vessel to ground. Because the plasma represents a variable load to the injector voltage, large voltage spikes well in excess of that applied can be produced. With the use of only $1 \mathrm{kV}$ on the CHI electrodes, voltage spikes $>3 \mathrm{kV}$ were measured which sometimes these resulted in external arcs. In order to reduce these voltage spikes analysis indicated that both $\mathrm{AC}$ and DC transient suppression is required. Transient suppression on the microsecond timescale is accomplished by use of a capacitive snubber circuit close to the vacuum vessel connected by low inductance leads. Suppression on longer timescales is provided by metal oxide varistors (MOV) connected in 4 parallel groups of 160 parallel modules. The current and voltage curve of the MOV assembly is shown in Fig. 3. This combination has limited the transient voltage spikes to $2.2 \mathrm{kV}$ with $\mathrm{V}_{\text {inj }}$ $=1.7 \mathrm{kV}$. In addition, because internal arcs in the absorber region at the top of the vessel, called absorber arcs, had previously resulted in the extinguishment of the desired discharge across the injector, the upper insulator region was redesigned. ${ }^{10}$ The upper ceramic ring was replaced by a much larger alumina insulator that was effective in reducing the incidence of absorber arcs and increased the impedance of any absorber arcs that did occur so that they did not cause termination of the desired discharge.

\section{MOV CHARACTERISTICS}

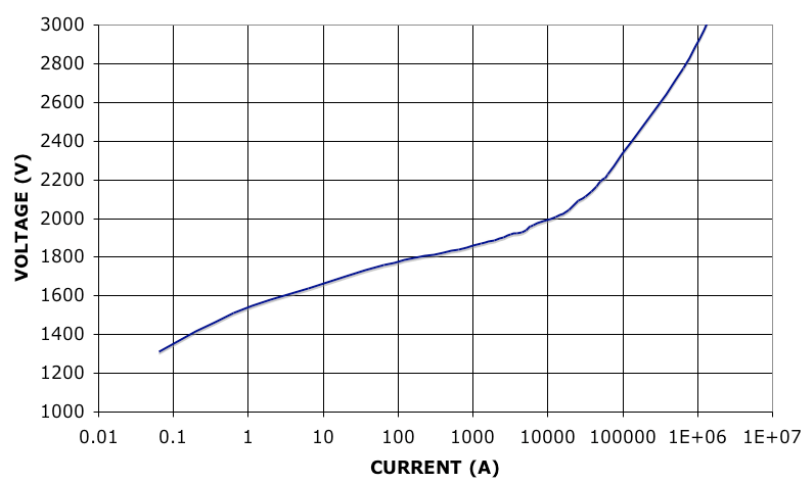

Fig. 3. The current through the $640 \mathrm{MOVs}$ connected in parallel is a non-linear function of the applied voltage. This assembly acts to limit the voltage as the plasma load impedance fluctuates.

The procedure to produce transient $\mathrm{CHI}$ discharges is as follows. The toroidal field current is ramped up to its desired value and the initial vacuum poloidal flux is supplied at $\mathrm{t}=0$. The pre-programmed amount of deuterium is then injected and the microwave preionization is applied in the volume below the lower divertor gap. The pre-charged capacitor bank is connected to the inner and outer vessel by an ignitron switch with the outer vessel acting as the anode to form a discharge, typically starting at $5 \mathrm{~ms}$. After a preprogrammed time (typically 3 to $5 \mathrm{~ms}$ ) chosen to allow the plasma to expand into the vessel volume and at a time near the peak in the toroidal current, the capacitor bank is shorted into a low resistance by a second ignitron. The injector current then falls rapidly to zero and the plasma detaches from the electrodes to form closed flux surfaces. Since there is no current flowing across the divertor gap while the toroidal plasma current persists for several ms, it is clear that the plasma current is flowing on closed field lines.
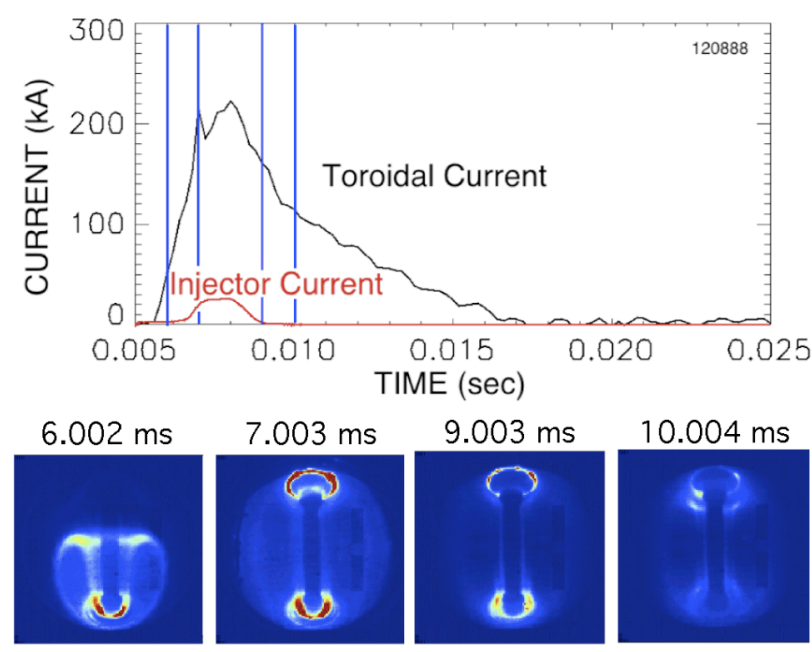

Fig. 4. The injector current is zero at $9 \mathrm{~ms}$ when the toroidal current is $160 \mathrm{kA}$. The 4 vertical lines in the top of the figure indicate the times of the 4 fast camera images from the same shot which are shown in the lower portion of the figure.

\section{EXPERIMENTAL RESULTS}

Traces of plasma current, injector current, and fast TV images for one of the highest current discharges that has toroidal current flowing only on closed field lines are shown in Figure 4. It is clear that the plasma grows to fill the vessel volume. In this case, as was the norm for the very highest current shots, an absorber arc occurred at the top of the machine, but the arc did not result in termination of the discharge. The current trace labeled injector current is actually the sum of the currents across the injector and the absorber gaps; the current is measured in the leads going to the vacuum vessel and there is no measurement of the current through the vacuum vessel. At about $9 \mathrm{~ms}$, when the injector current falls to zero, $\mathrm{I}_{\mathrm{p}}=$ $160 \mathrm{kA}$ and after that the plasma decays resistively. It is worth noting that the poloidal coil currents varied only slightly during the plasma duration to center the plasma vertically and the ohmic solenoid was not used at all. 


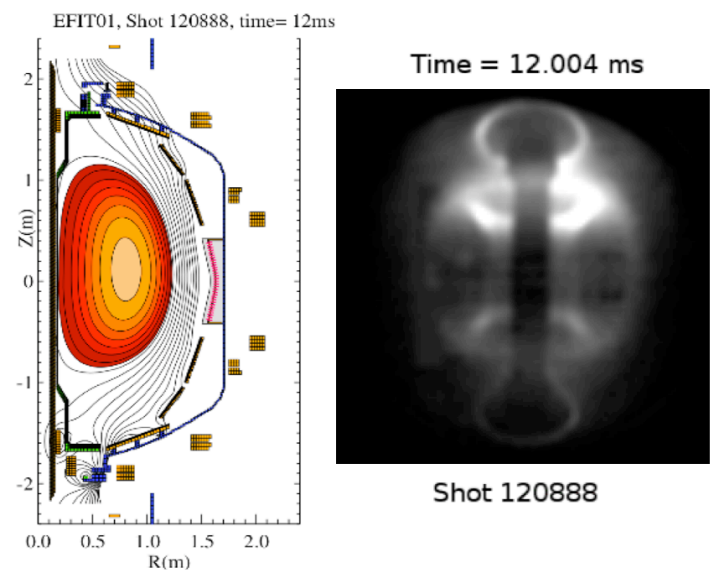

Fig. 5. Flux plot from EFIT analysis compared to fast camera image at the same time.

After the plasma becomes detached from the injector, the plasma current decays to near zero in about 8 ms. During this decay phase when there is no current flowing on open field lines, the EFIT code ${ }^{11}$ can be used to analyze the magnetic data. Fig. 5 shows a flux plot at $12 \mathrm{~ms}$ during the current decay phase, $3 \mathrm{~ms}$ after $\mathrm{I}_{\mathrm{inj}}=0$, with a fast camera image from the same time. There is respectable agreement between the flux plot and shape of the plasma image. The electron temperature and density profiles were measured by Thomson scattering. The Thomson system on NSTX employs 2 lasers that each
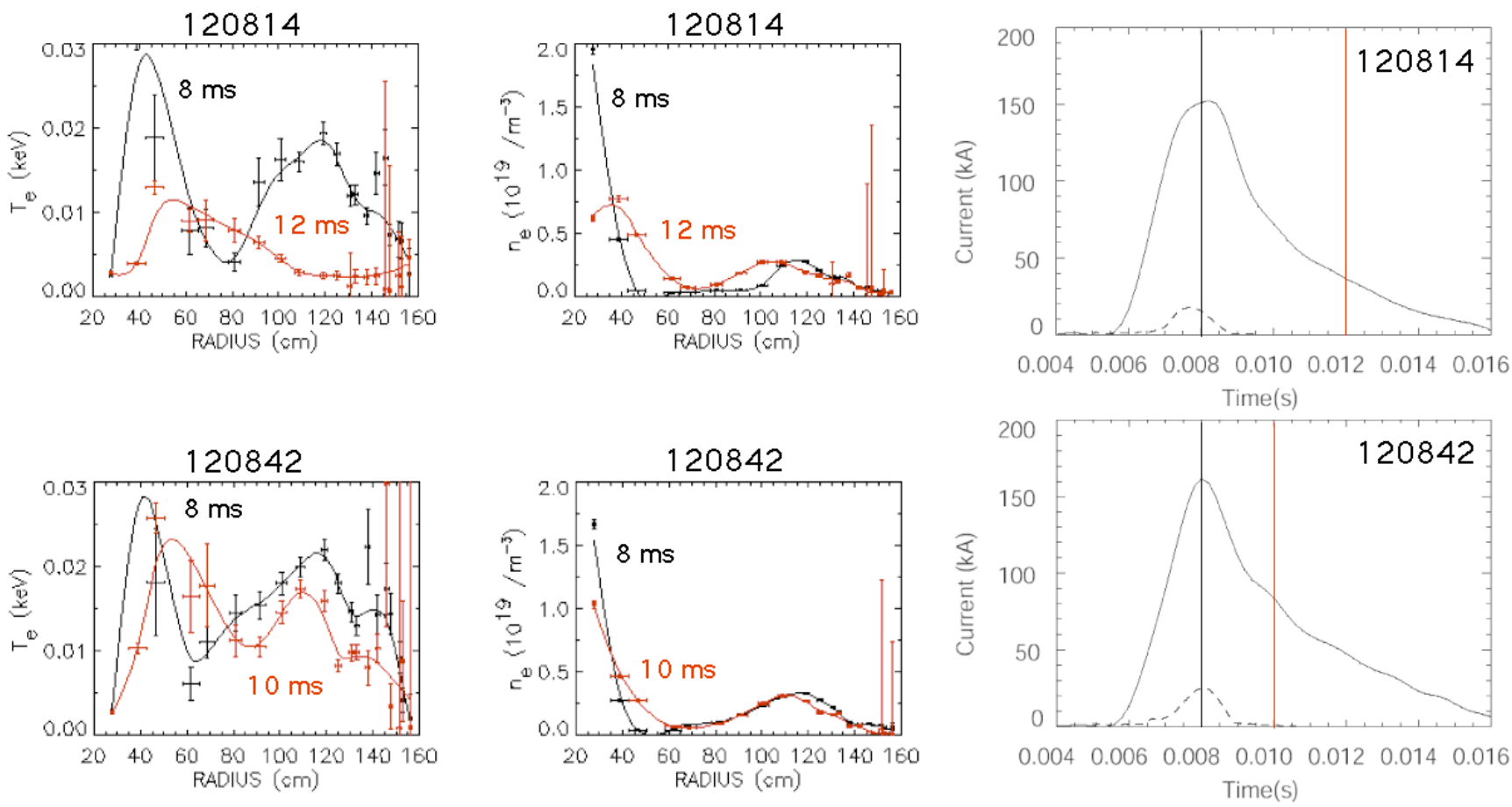

Fig. 6. Electron temperature and density profiles from Thomson scattering measurements at the peak of the current and during the current decay phase. Vertical bars in the current vs time plots indicate the Thomson times. 
this positive scaling, however, the 6 conditions discussed in section II must be met. Of these conditions, most can be met by appropriate choices of gas fueling, capacitor size and charging voltage. As the size of the device increases, the connection length between the electrodes will increase, so $\mathrm{V}_{\text {inj }}$ required to produce the discharge will rise. Voltage standoff considerations will eventually place a limit on the amount of current that can be produced. For example, a 1 MA ST discharge with $\mathrm{L}_{\mathrm{p}}=$ $0.5 \mu \mathrm{H}$ would have a magnetic stored energy of $250 \mathrm{~kJ}$, less than half the energy that could be supplied by a 50 $\mathrm{mF}$ capacitor charged to $5 \mathrm{kV}$. Such a capacitor would easily supply the 50 Coulomb charge needed for a $5 \mathrm{kA}$, $10 \mathrm{~ms}$ duration injector current, which would be more than enough to meet the bubble burst condition. The connection length from one electrode to the other, however, increases as $\psi_{\mathrm{T}} / \psi_{\text {inj }}$, which, assuming the same resistivity, requires a $\mathrm{V}_{\text {inj }} / \mathrm{I}_{\text {inj }}$ to increase proportionally. Taken together these imply that the achievable $I_{p}$ will scale as $\mathrm{V}_{\text {inj }}$, which has practical limits.

Finally, in order to ramp $I_{p}$ further with other means, it may be necessary to heat the discharge above the presently observed $20 \mathrm{eV}$ to a few hundred $\mathrm{eV}$ in order to improve coupling to RF current drive techniques.

\section{SUMMARY}

Using the method of transient CHI, world-record non-inductively start-up plasma current flowing on closed field lines in a toroidal confinement device of $160 \mathrm{kA}$ has been unambiguously achieved. The resulting plasmas have electron temperatures of about $20 \mathrm{eV}$ and decay resistively in several ms. Planned future experiments will include study of the scaling of the start-up current with injection voltage, toroidal field and the initial poloidal flux pattern.

\section{ACKNOWLEDGMENTS}

We gratefully acknowledge the NSTX team especially E. Fredd, R. Hatcher, S. Ramakrishnan and C. Neumeyer without whose support these experiments would have been impossible. This work supported by US DOE Contract Nos. DE-AC02-76CH03073 and DEFG03-96ER54361.

\section{REFERENCE}

1. Y-K M. PENG, "A component test facility based on the spherical tokamak," Plas. Phys. Cont. Fus., 47 B263 (2005)

2. C.W. BARNES et al., "Experimental determination of the conservation of magnetic helicity from the balance between source and spheremak," Phys. Fluids, 29, 3415 (1986).

3. M. ONO, et al., "Exploration of spherical torus physics in the NSTX," Nucl. Fusion, 40, 3Y 557 (2000).

4. J.E. MENARD, et al., "Recent Physics Results from NSTX," Proc. 21st IAEA Fus. Eng. Conf. (Chengdu, China, Oct. 2006) Plasma Phys. Cont. Fus.

5. R. RAMAN, et al., "Non-inductive current generation in NSTX using coaxial helicity injection," Nucl. Fus., 41, 1081 (2001).

6. B.A. NELSON, et al., "Formation and sustainment of a low-aspect ratio tokamak by coaxial helicity injection," Phys. Plas., 2, 2337 (1995).

7. A.J. REDD, et al., "Current drive experiments in the helicity injected torus (HIT-II)," Phys. of Plas., 9, 2006 (2002).

8. R. RAMAN, et al., "Experimental demonstration of plasma startup by coaxial Helicity injection," Phys. of Plas., 11, 2565 (2004).

9. T.R. JARBOE, "Formation and steady state sustainment of a tokamak by coaxial helicity injection." Fus. Technol., 15, 7 (1989).

10. D. MUELLER, et al., "Design, installation and performance of the new insulator for NSTX CHI experiments," 21st IEEE/NPSS SOFE2005, IEEE Cat. No. 05CH37764C Knoxville, Tennessee, 26-29 September 2005.

11. L.L. LAO, et al., "Reconstruction of current profile parameters and plasma shapes in tokamaks." $\mathrm{Nucl}$. Fus , 25,1611 (1985)

S.A. SABBAGH, et al., "Equilibrium properties of spherical torus plasmas in NSTX," Nucl. Fus., 41, 1601 (2001). 